\title{
On the existence and uniqueness of solutions of parabolic equations
}

\section{K.L. Teo}

Recently, Ekl und (Proc. Amer. Math. Soc. 47 (1975), 137-142) has shown that to each continuous function $F$ on $\partial_{p} Q \leq\{\partial \Omega \times[0, T]\} \cup\{\Omega \times(0)\}$ there is a unique solution to the boundary value problem

$$
\left\{\begin{array}{cl}
(L \phi)(x, t) & =0 \quad, \quad(x, t) \in \Omega \times(0, T], \\
\phi(x, t) & =F(x, t),(x, t) \in \partial_{p^{Q}},
\end{array}\right.
$$

where $L$ is a linear second order parabolic operator in divergence form, $\Omega \subset R^{n}$ is a bounded domain with compact closure and $\partial \Omega$ denotes its boundary. In this note, it is shown that the existence theorem of EkI und remains valid for the following boundary problem

$$
\left\{\begin{aligned}
(L \phi)(x, t) & =f(x, t)+\left(g_{i}(x, t)\right)_{x_{i}}, \quad(x, t) \in \Omega \times(0, T], \\
\phi(x, t) & =F(x, t)
\end{aligned}\right.
$$

1. Introduction and preliminary results

Let $\Omega \subset R^{n}$ be open with compact closure $\bar{\Omega}=\Omega \cup \partial \Omega$. Let $T>0$ and $Q \leq \Omega \times(0, T]$. Further, let $\partial_{p} Q \leq\{\partial \Omega \times[0, T]\} \cup\{\Omega \times(0)\}$ denote the parabolic boundary of $Q$.

Consider the system described by the following linear second order partial differential equation of parabolic type with non-homogeneous

Received 10 september 1975. 
boundary conditions:

$$
S:\left\{\begin{aligned}
(L \phi)(x, t)=f(x, t)+\left(g_{i}(x, t)\right)_{x_{i}}, & (x, t) \in Q, \\
\phi(x, t)=F(x, t) & , \quad(x, t) \in \partial_{p} Q,
\end{aligned}\right.
$$

where the (parabolic) differential operator $L$ is given by

$$
L u \unlhd u_{t}-\left\{a_{i j}(x, t) u_{x_{i}}+a_{j}(x, t) u\right\}_{x_{j}}-b_{j}(x, t) u_{x_{j}}-c(x, t) u
$$

with

$$
\psi_{t} \unlhd \frac{\partial \psi}{\partial t}, \quad \psi_{x_{j}} \unlhd \frac{\partial \psi}{\partial x_{j}} \text {, and } \psi_{x_{i} x_{j}} \leq \frac{\partial^{2} \psi}{\partial x_{i} \partial x_{j}} \text {. }
$$

Note that the convention adopted above and throughout the rest of the note is to take summation up to $n$ over repeated indices.

The given functions and solutions will lie in multidimensional $L^{p}$ spaces and Sobolev space $L^{2}\left[0, T, H^{1}, 2(\Omega)\right]$. These spaces are defined in detail by Aronson and Serrin [2].

Throughout the note, the coefficients of $L$ are assumed to satisfy the following assumptions which will be referred to collectively as (A).

(i) The $a_{i j}(x, t)$ are measurable functions in $(x, t)$ with $\left|a_{i j}(x, t)\right| \leq M<\infty$ almost everywhere in $Q$, and for some $\lambda>0$, $a_{i j}(x, t) z_{i} z_{j} \geq \lambda|z|^{2}=\lambda \sum_{i=1}^{n} z_{i}^{2}$ for all $z \in R^{n}$ and almost all $(x, t) \in Q$.

(ii) $c(x, t) \in L^{q}\left[0, T ; L^{p}(\Omega)\right]$ for some pair $p$, $q$ satisfying

$$
1<p, q \leq \infty,
$$$$
n / 2 p+1 / q<1 \text {. }
$$

(iii) $b_{j}(x, t), a_{j}(x, t) \in L^{q}\left[0, T, L^{p}(\Omega)\right]$ for some pair $p, q$ satisfying

$$
2<p, q \leq 0,
$$




$$
n / 2 p+1 / q<\frac{1}{2}
$$

For convenience of reference, one basic definition and two basic theorems of Aronson [1], and the main theorem of Ek/und [3] are quoted without proof.

DEFINITION 1. Let $L$ be as described above, $f(x, t) \in L^{q}\left[0, T ; L^{p}(\Omega)\right]$ where $p, q$ satisfy $(*)$ and $g_{i}(x, t) \in L^{q}\left[0, T ; L^{p}(\Omega)\right]$ where $p, q$ satisfy $(* *)$. Then, $u(x, t)$ is said to be a weak solution of the boundary value problem

$$
\left\{\begin{aligned}
(L u)(x, t) & =f(x, t)+\left\{g_{i}(x, t)\right\}_{x_{i}}, & & (x, t) \in Q, \\
u(x, t) & =0 & & (x, t) \in S \leq \partial \Omega \times[0, T], \\
u(x, 0) & =u_{0}(x) & , & x \in \Omega,
\end{aligned}\right.
$$

if

$$
\begin{aligned}
& \text { (i) } u \in L^{2}\left[\delta, T ; H_{10 c}^{1,2}(\Omega)\right] \cap L^{\infty}\left[\delta, T ; L_{10 c}^{2}(\Omega)\right] \text { for each } \delta>0 \text {, } \\
& \text { (ii) } u_{0}(x) \in L^{2}(\Omega)
\end{aligned}
$$

and if, for each $z(x, t) \in C^{\mathcal{I}}(\bar{Q})$ with compact support in $\Omega$,

$$
\text { (iii) } \begin{aligned}
\int_{Q}\left[a_{i j} x_{i}{ }^{z} x_{j}{ }^{+a}{ }_{j}^{z} x_{j}^{u-b}{ }_{j}^{u} x_{j}^{z-c u z-u z} t\right] d x d t \\
=\int_{Q}\left[f z-g_{i} x_{i}\right] d x d t+\int_{\Omega} u_{0}(x) z(x, 0) d x,
\end{aligned}
$$

and

$$
\text { (iv) } \lim _{t \downarrow 0} \int_{\Omega} u(x, t) z(x, t) d x=\int_{\Omega} u_{0}(x) z(x, 0) d x \text {. }
$$

Aronson and Serrin [2] have shown that every weak solution of (1) in $Q$ has a representative that is continuous in $Q$. Henceforth, $u$ will denote the continuous representative of a given weak solution.

THEOREM A. Suppose that the assumption (A) is satisfied and that $u_{0}(x), f(x, t), g_{i}(x, t)$ are as described in Definition 1 . Then there is a unique weak solution $u$ of the boundary value problem (I). 
A proof of this theorem is given by Aronson in [1].

THEOREM B. Let $u$ be a solution of the problem (1) with $f \equiv 0$, $g_{i} \equiv 0$ on $Q$, and $u_{0} \in L^{2}(\Omega)$. Further, let $\zeta=\zeta(x)$ be a nonnegative smooth function such that $\zeta u \in L^{2}\left[0, T, H_{0}^{1,2}(\Omega)\right]$. Then there is a positive constant $C$ such that

$$
\|\zeta u\|_{2, \infty}^{2}+\left\|\zeta u_{x}\right\|_{2,2}^{2} \leq c\left\{\int_{\Omega} \zeta^{2} u_{0}^{2} d x+\left\|\zeta_{x} u\right\|_{2,2}^{2}\right\} \text {. }
$$

A proof of this theorem can be found in [1].

THEOREM C. Suppose that the assumption (A) is satisfied and that $F(x, t)$ is continuous on $S$ so that $F(x, 0) \in L^{2}(\Omega)$. Then there is a unique weak solution $u$ of the boundary value problem

$$
\left\{\begin{aligned}
(L u)(x, t) & =0, & (x, t) \in Q, \\
u(x, t) & =F(x, t), & (x, t) \in \partial_{p} Q .
\end{aligned}\right.
$$

A proof of this theorem is given by Eklund in [3].

\section{Main result}

We are now to present our main result in the following theorem. Its proof is based on Theorems (A), (B), and (C) given in the previous section.

THEOREM 1. Consider the boundary value problem (S). Suppose that the assumption (A) is satisfied and that $F(\cdot, \cdot)$ is continuous on $S$ and $F(\cdot, 0) \in L^{2}(\Omega)$. Then there is a unique weak solution $u$ of the problem (S).

Proof. In view of Theorems (A) and (C), we note that the boundary value problems (I) and (2) possess unique solutions $u$ and $v$ respectively. Define

$$
\psi \leq u+v
$$

We shall show that $\psi$ is the unique weak solution of the problem (S). First of all, we see that for each $z \in C^{\mathcal{I}}(\bar{Q})$ with compact support in $\Omega$, 


$$
\begin{aligned}
\int_{Q}(L \psi) z d x d t & =\int_{Q}(L u) z d x d t+\int_{Q}(L v) z d x d t \\
& =\int_{Q}\left[f z-g_{i} x_{i}\right] d x d t+\int_{\Omega} F(x, 0) z(x, 0) d x
\end{aligned}
$$

Further,

$$
\begin{aligned}
\lim _{t \downarrow 0} \int_{\Omega} \psi(x, t) z(x, t) d x & =\lim _{t \downarrow 0}\left\{\int_{\Omega} u(x, t) z(x, t) d x+\int_{\Omega} v(x, t) z(x, t) d x\right\} \\
& =\lim _{t \downarrow 0} \int_{\Omega} u(x, t) z(x, t) d x+\lim _{t \downarrow 0} \int_{\Omega} v(x, t) z(x, t) d x \\
& =\int_{\Omega} F(x, 0) z(x, 0) d x .
\end{aligned}
$$

This implies that $\psi$ is a weak solution of the problem (S).

It remains to show the uniqueness. For this, we assume that $u_{1}$ and $u_{2}$ are two distinct solutions of the problem (s). Then, define

$$
\phi \leq u_{1}-u_{2}
$$

and consider the following boundary value problem

$$
\left\{\begin{aligned}
(L \phi)(x, t)=0, & (x, t) \in Q, \\
\phi(x, t)=0, & (x, t) \in \partial_{p} Q .
\end{aligned}\right.
$$

Therefore, it follows from Theorems (A) and (B) that $\phi$ is also the unique solution of the problem ( 3 ) and is equal to zero identically on $\bar{Q}$. This simply implies that $u_{1} \equiv u_{2}$ on $\bar{Q}$. Thus, we can conclude that the problem (S) admits only a unique weak solution $\psi$. This completes the proof.

\section{References}

[1] D.G. Aronson, "Non-negative solutions of linear parabolic equations", Ann. Scuola Norm. Sup. Pisa 22 (1968), 607-694.

[2] D.G. Aronson and James Serrin, "Local behavior of solutions of quasilinear parabolic equations", Arch. Rational Mech. Anal. 25 (1967), 81-122. 
[3] Neil A. Eklund, "Existence and representation of solutions of parabolic equations", Proc. Amer. Math. Soc. 47 (1975), 137-142.

[4] Avner Friedman, Partial differential equations of parabolic type (Prentice-Hall, Englewood Cliffs, New Jersey, 1964).

[5] O.A. Ladyženskaja, V.A. Solonnikov, N.N. Ural'ceva, Linear and quasiZinear equations of parabolic type (Translations of Mathematical Monographs, 23. Amer. Math. Soc., Providence, Rhode Island, 1968).

School of Mathematics,

University of New South Wales,

Kensington,

New South Wales. 